\title{
Performance and Reporting Channel Traffic of Eigenvalue Fusion and Block-coded Decision Fusion for Spectrum Sensing of OFDMA Signals
}

\author{
Lucas dos Santos Costa, Dayan Adionel Guimarães and Rausley Adriano Amaral de Souza, Member, IEEE
}

\begin{abstract}
This paper presents a comparison between performance and data traffic in the reporting control channel of a cooperative centralized spectrum sensing scheme designed to detect idle OFDMA subchannels for cognitive radio applications. Eigenvalue and decision fusion strategies are compared when repetition and $\mathrm{BCH}$ block codes are used to protect the data carrying the sensor decisions. It is unveiled that the potential larger sensitivity to channel errors of the decision fusion can be supplanted when block codes are used, in some cases leading to an overall smaller amount of traffic when compared with the eigenvalue fusion. However, the advantage of the decision fusion over the eigenvalue fusion is not always pronounced, and sometimes reversed, demanding a case-by-case trade-off analysis regarding the target performance and the reporting channel traffic.
\end{abstract}

Index Terms-Cognitive radio, eigenvalue fusion, ED, GLRT, MED, MMED, OFDMA, spectrum sensing.

\section{INTRODUCTION}

$\mathbf{T}$ He present demand for wireless communications services and the fixed allocation policy of the radio-frequency spectrum have produced scarcity and congestion of frequency bands on an unprecedented way. The cognitive radio (CR) concept [2] has come as a promising solution to this problem, by allowing the opportunistic use of idle bands by the CRs that compose a secondary network. To this end, a spectrum sensing [3] process must take place, combined or not with some database information, aiming at finding the so-called spectral holes or white-spaces. Subsequent access to the whitespaces is performed, keeping the level of interference to the primary users (which owns the rights of using that frequency band) below a maximum acceptable level.

It is then undeniable the importance of the research efforts directed towards spectrum sensing techniques. These efforts are now even more motivated by the recent creation of

The Associate Editors coordinating the review of this manuscript and approving it for publication were Prof. Cecílio José Lins Pimentel and Prof. Marcelo da Silva Pinho.

Lucas dos S. Costa, Dayan A. Guimarães and Rausley A. A. de Souza are with National Institute of Telecommunications (Instituto Nacional de Telecomunicações - Inatel), Santa Rita do Sapucaí - MG, Brazil, +55 3471 9227, e-mails: $\{$ lucass,dayan,rausley\}@inatel.br.

This work was partially supported by Finep, with resources from Funttel, Grant No. 01.14.0231.00, under the Radiocommunication Reference Center (Centro de Referência em Radiocomunicações - CRR) project of the National Institute of Telecommunications (Instituto Nacional de Telecomunicações Inatel), Brazil.

A preliminary version of this paper was presented in XXXIII Simpósio Brasileiro de Telecomunicações (SBrT'15), Juiz de Fora, MG, Brazil, September 1-4, 2015 [1].

Digital Object Identifier: 10.14209/jcis.2016.4 the 802.22.3 Task Group on Spectrum Characterization and Occupancy Sensing (SCOS) [4] intending to enable broadband wireless access using cognitive radio technology and spectrum sharing in white spaces. As stated by the chair of the group, "standardization could lead to the more efficient use of spectrum, especially in places where the information about the primary users is difficult to find". Yet, "individual and collaborative spectrum sensing is one of the tools to complement the information contained in databases to create an accurate spectrum occupancy survey, which would combine information from multiple sensors along with local terrain information to predict the spectrum occupancy patterns" [4].

The majority of third-generation $(3 \mathrm{G})$ wideband communication systems are based on direct-sequence spread spectrum (DSSS), whereas in the fourth-generation (4G) it is prevalent the use of multicarrier techniques, such as the orthogonal frequency division multiplexing (OFDM), combined or not with its multiple access counterpart, the orthogonal frequency division multiple access (OFDMA). The use of OFDM is justified from its advantages in delivering high data rates, especially over frequency-selective multipath fading channels, and from its ability in generating null subcarriers. This last advantage is of particular interest to the opportunistic access to non-contiguous idle frequency bands, which seems to be a more realistic scenario since it is hard to find large continuous idle bands in practice. The generalized frequency division multiplexing (GFDM) [5] has come as a generalization of the OFDM and a strong candidate waveform for the fifthgeneration $(5 \mathrm{G})$ systems. Among its advantages is the flexible way of generating transmission waveforms in a noncontiguous spectrum, with an out-of-band emission way below the one achieved with the traditional OFDM waveform.

The wideband spectrum sensing techniques aim at detecting the primary signal in multiple bands simultaneously or sequentially, in contrast with narrowband spectrum sensing, which monitors small portions of the spectrum. Typical techniques adopted in narrowband spectrum sensing are the energy detection, the matched filter detection, and the cyclostationary feature detection [3]. In the case of wideband signals, the energy detection can be used as well, but more elaborate techniques are preferred, as for example the wavelet-based detection and the compressive (or compressed) sensing [6]. The eigenvalue-based detection [7] techniques are the most recent and one of the most promising solutions to the problem of spectrum sensing of both narrowband and wideband signals.

No matter if in its narrowband or wideband form, the 
spectrum sensing can be performed independently by each $\mathrm{CR}$, or can resort to the sensing information coming from multiple CRs. The former is simpler, but carriers the intrinsic disadvantage of being less reliable, mainly due to receiver uncertainty, multipath fading and shadowed signals. On the other hand, the later, which is usually referred to as cooperative or collaborative spectrum sensing, is more complex but renders a more reliable decision upon the occupation state of the sensed band, simultaneously combating the receiver uncertainty, the multipath fading and the shadowing effects [3]. Cooperative spectrum sensing can be centralized, distributed or relay-assisted. In the centralized cooperative spectrum sensing, which is the focus of this paper, data (e.g. received signal samples) collected by each $\mathrm{CR}$ is sent to a fusion center (FC) through dedicated reporting channels, configuring what is called data fusion scheme. The centralized cooperative spectrum sensing can also resort to the CRs' decisions about the channel occupation state, which is known as decision fusion (DF) strategy. In this case the FC combines the CRs' decisions using some binary operation, as for instance the general $k$-out-of- $n$ operation from where are derived the wellknown AND, OR and majority-voting (MAJ) rules. In both centralized schemes the final decision upon the occupation of the channel is made at the FC and informed back to the CRs for subsequent opportunistic channel access.

A new approach for detecting OFDMA and other wideband signals was proposed in [8], in the context of cooperative centralized data fusion spectrum sensing. In this approach, the detection is based on the eigenvalues of the received signal covariance matrix whose samples are in the frequency domain. The main novelty in [8] is that the eigenvalues are computed at the CRs and sent to the FC, where they are combined. The new technique has been named eigenvalue fusion (EVF). In [8], four test statistics were assessed, namely: the generalized likelihood ratio test (GLRT), the maximum-minimum eigenvalue detection (MMED), also know as eigenvalue ratio detection (ERD), the maximum eigenvalue detection (MED), sometimes referred to as the Roy's largest root test (RLRT), and the energy detection (ED). The results unveiled that the EVF can achieve better performance when compared with the decision fusion and sample fusion schemes, in terms of the probabilities of false alarm and detection. Moreover, it has been shown in [8] that the EVF produces less data traffic in the reporting control channel when compared with the sample fusion; the decision fusion is intrinsically the strategy that produces less reporting traffic.

An analysis about the spectrum sensing performance and the volume of data over the erroneous reporting channels is presented in [9], considering the EVF and the DF schemes and repetition-coded decisions in the DF scheme, also in the context of sensing OFDMA subchannels. The study was motivated by a conjecture in [8], stating that errors in the reporting channels could be more disastrous to the bits representing CRs' decisions in the DF than to the bits representing the eigenvalues in the EVF. This would demand more protection to the CRs' decisions, reducing the difference between the volume of the reporting traffic for the DF and the EVF, putting the EVF in a better position both in terms of performance and number of bits sent to the FC. The results in [9] have demonstrated that such a conjecture is partially true: CRs' decisions are indeed more sensitive to channels errors, but the amount of redundancy added to equate the performances of the EVF and the coded DF does not always render a larger number of bits sent to the FC in the case of the DF. As a consequence, a trade-off solution should be established.

Other more powerful coding schemes could be used instead of the repetition codes, bettering even more the advantage of the DF with respect to the EVF. Moreover, the use of other block codes would avoid the performance degradation caused by repetition encoding when the fading in the reporting channels becomes less severe or exhibits high time correlation. To this end, one option would be to apply block codes with message length equal to the number of OFDMA subchannels. This paper deals with this solution, considering repetition and Bose-Chaudhuri-Hocquenghem $(\mathrm{BCH})$ codes. A through analysis is made by considering these codes with different coding rates applied to the DF scheme, resulting an extensive comparison with the EVF for the detection techniques GLRT, MMED, MED and ED, and for the DF combining rules AND, OR and MAJ. This paper complements and extends the results of the conference paper [1], where only the GLRT is analyzed in detail.

The remaining of this paper is organized as follows: Section II, which is grounded on [8], concisely reviews the operation of the eigenvalue fusion and the decision fusion for detecting idle OFDMA subchannels. Section III presents the system model. Plenty of numerical results and discussions are provided in Section IV. Section V concludes the paper and gives some directions for future research on the topic.

\section{OFDMA SUBCHANNEL DETECTION WITH EVF AND DF}

In the OFDMA, a set or multiple sets of subcarriers are assigned to a given user, allowing for the simultaneous access by several users to the whole available bandwidth. This set of subcarriers is usually referred to as an OFDMA subchannel. It can be formed under the adjacent subcarrier method (ASM), or using non-adjacent subcarriers in the diversity subcarrier method (DSM). Then, the spectrum sensing of OFDMA signals is made at the subchannel level, that is, the goal is to seek for one or more idle subchannels.

Let an OFDMA signal with $P$ subchannels each indexed by $s, s=1,2, \ldots, P$, and a total of $K$ subcarriers. A number $K^{\prime}=K / P$ subcarriers will form a subchannel. It is assumed that the $m$ cooperating CRs have knowledge of the allocation map of the $P$ subchannels, an information that is readily available from the knowledge of the primary network standard. A matrix of order $K^{\prime} \times N$ with sample values of the received signal by the $i$-th $\mathrm{CR}$ in the $s$-th subchannel is formed according to

$$
\mathbf{A}_{s}^{(i)}=\left[\begin{array}{ccc}
Y_{1,1}^{(i)}(s) & \cdots & Y_{1, N}^{(i)}(s) \\
\vdots & \ddots & \vdots \\
Y_{K^{\prime}, 1}^{(i)}(s) & \cdots & Y_{K^{\prime}, N}^{(i)}(s)
\end{array}\right]
$$

with $N$ being the number of samples collected by each $\mathrm{CR}$ in each subcarrier of a given subchannel, and $Y_{k^{\prime}, j}^{(i)}(s)$ denotes 
the $j$-th sample collected by the $i$-th CR in the $k^{\prime}$-th subcarrier of the $s$-th subchannel, for $j=1,2, \ldots, N, i=1,2, \ldots, m$ and $k^{\prime}=1,2, \ldots, K^{\prime}$.

The next step consists of computing the corresponding sample covariance matrices of the received signals as

$$
\mathbf{R}_{s}^{(i)}=\frac{1}{N} \mathbf{A}_{s}^{(i)} \mathbf{A}_{s}^{(i)}{ }^{\dagger}
$$

where $\dagger$ stands for complex conjugate and transpose. These covariance matrices are operated in the EVF and DF schemes as follows.

\section{A. EVF for OFDMA signals}

From (2), $K^{\prime} P$ eigenvalues are computed by each $\mathrm{CR}$ and the resulting $m K^{\prime} P$ eigenvalues are sent to the $\mathrm{FC}$ in the EVF scheme. The test statistic associated to the $s$-th OFDMA subchannel is formed at the FC from one amongst the following expressions [8]:

$$
\begin{array}{r}
T_{\mathrm{GLRT}, s}=\frac{P K^{\prime} \sum_{i=1}^{m} \lambda_{1, s, i}}{\sum_{j=1}^{K^{\prime}} \sum_{z=1}^{P} \sum_{i=1}^{m} \lambda_{j, z, i}} \\
T_{\mathrm{MED}, s}=\frac{\sum_{i=1}^{m} \lambda_{1, s, i}}{m \sigma^{2}} \\
T_{\mathrm{MMED}, s}=\frac{P \sum_{i=1}^{m} \lambda_{1, s, i}}{\sum_{z=1}^{P} \sum_{i=1}^{m} \lambda_{K^{\prime}, z, i}} \\
T_{\mathrm{ED}, s}=\frac{\sum_{j=1}^{K^{\prime}} \sum_{i=1}^{m} \lambda_{j, s, i}}{K^{\prime} m \sigma^{2}}
\end{array}
$$

where $\left\{\lambda_{1, s, i} \geq \lambda_{2, s, i} \geq \cdots \lambda_{K^{\prime}, s, i}\right\}$ are the $K^{\prime}$ ordered eigenvalues associated to the $s$-th subchannel at the $i$-th CR, and $\sigma^{2}$ is the variance of the zero-mean white Gaussian noise at the input of each $\mathrm{CR}$.

\section{B. DF for OFDMA signals}

For the DF scheme, matrices with received signal samples for each $\mathrm{CR}$ and each subchannel are formed according to (1), and the corresponding sample covariance matrices are calculated from (2). For each matrix among the $P$ matrices computed from (2), each CR will compute $K^{\prime}$ eigenvalues $\left\{\lambda_{1, s} \geq \lambda_{2, s} \geq \cdots \lambda_{K^{\prime}, s}\right\}$. The states of occupation of the subchannels are determined in each CR by comparing one among the following test statistics [8] with a decision threshold:

$$
\begin{gathered}
T_{\mathrm{GLRT}, s}=\frac{P K^{\prime} \lambda_{1, s}}{\sum_{j=1}^{K^{\prime}} \sum_{z=1}^{P} \lambda_{j, z}} \\
T_{\mathrm{MED}, s}=\frac{\lambda_{1, s}}{\sigma^{2}} \\
T_{\mathrm{MMED}, s}=\frac{P \lambda_{1, s}}{\sum_{z=1}^{P} \lambda_{K^{\prime}, z}} \\
T_{\mathrm{ED}, s}=\frac{\sum_{j=1}^{K^{\prime}} \lambda_{j, s}}{K^{\prime} \sigma^{2}} .
\end{gathered}
$$

The $m P$ CRs' decisions are then sent to the $\mathrm{FC}$ for subsequent binary logic operation (OR, AND or MAJ) and final decision upon the occupation of each subchannel.

\section{SYSTEM MODEL}

The uncoded EVF and coded DF schemes follow the pictorial models shown in Figs. 1 and 2, respectively.

In the case of the EVF, no channel coding was applied. Instead, the eigenvalues computed in each $\mathrm{CR}$ were digitized by the analog-digital converter (ADC) with a $b$-bit resolution and sent through the binary symmetric channels (BSC). At the FC, the corrupted received bits were converted into analog quantities by the digital-analog converter (DAC), thus representing corrupted eigenvalues. These eigenvalues were then combined according to the desired test statistic chosen from (3)-(6), and the final decisions upon the sensed subchannels were made.

In order to analyze the influence in the performance of the spectrum sensing caused by errors in the reporting channels from the CRs to the FC in the case of the DF, the

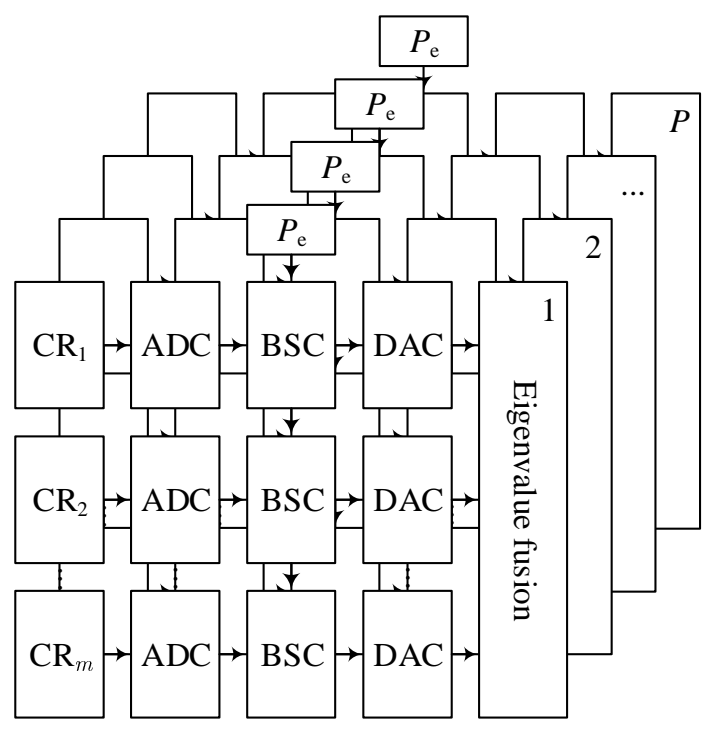

Fig. 1: Eigenvalue fusion (EVF) diagram for detecting OFDMA subchannels.

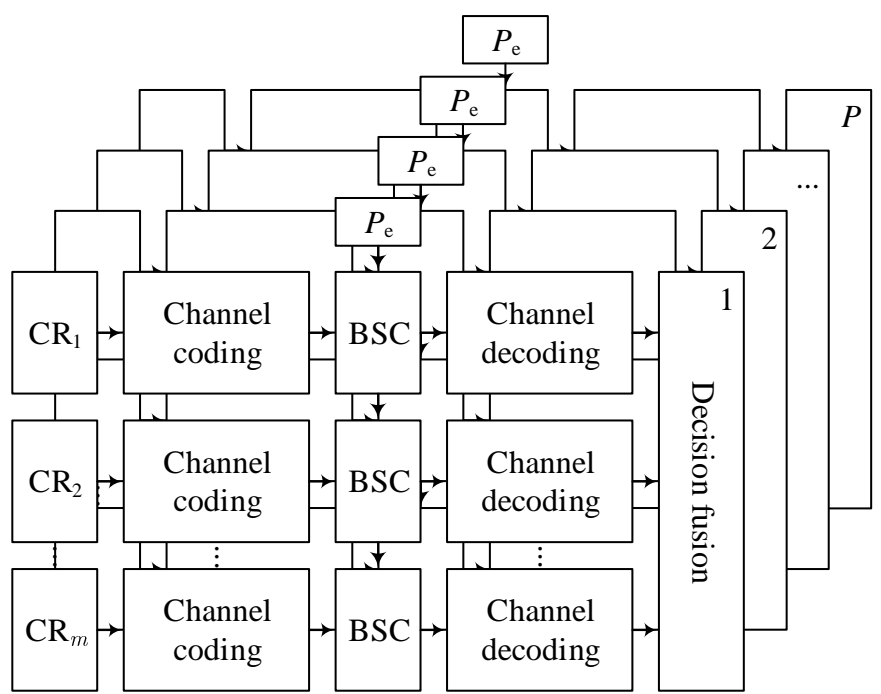

Fig. 2: Decision fusion (DF) diagram for detecting OFDMA subchannels. 
decisions made by each $\mathrm{CR}$ regarding the occupation of the sensed subchannels were subjected to block channel encoding, considering the local (at the CRs) test statistics GLRT, MMED, MED and ED, according to (7)-(10). Repetition codes with configurable coding rate $r=1 / n$, odd $n$, and $\mathrm{BCH}(n, k, t)$ coded were used, with message block length $k=P$, codeword length $n$ and error correction capability $t$ bits. After encoded, the decisions of each CR were sent through a memoryless BSC with configurable crossover (error) probability, $P_{\mathrm{e}}$. In the case of repetition encoding, the decoding was made via the majority rule. In the case of $\mathrm{BCH}$ encoding, decoding was made via the Berlekamp algorithm wicker1995error,berlekamp1968algebraic, operating with hard decisions. For both cases, the decoded CRs' decisions were combined according to the AND, the OR or the MAJ rule, and the final decisions on the occupancy of the sensed subchannels were reached.

Without loss of generality, the memoryless BSC channel model has been used to make it easy the configuration of the error probabilities and the analysis of the results. This channel can represent both the additive Gaussian noise (AWGN) and the AWGN plus fading reporting channels with interleaving depth enough to comply with the memoryless property of the model. The repetition code, the simplest among the channel codes, has been chosen due to the easy control of the coding rate and, thus, of the error correction capability. This brings flexibility in terms of the amount of redundancy inserted for a target performance, which is particularly favorable to the analysis made in this paper. One must recall that repetition codes are trivial, that is, they are not capable of producing real coding gains, but can produce equivalent performance improvements via diversity gains when fading reporting channels are considered. The $\mathrm{BCH}$ codes has been also considered not only for being superior to the repetition codes, producing real coding gains, but also to reduce the volume of traffic over the reporting channels in the DF scheme when compared with the use of the repetition codes. This choice brings even more advantage of the DF over the EVF if only the number of bits sent to the FC is under analysis (recall that the EVF is superior in performance to the DF scheme).

\section{NUMERICAL RESULTS}

The parameters adopted for the generation and analysis of the numerical results presented in this section corresponds to an OFDMA-based primary network with $K=20,25,30,40$ and 75 subcarriers, $P=4,5,6,8$ and 15 subchannels, respectively, and $m=6 \mathrm{CRs}$ in cooperation. The number of subcarriers per subchannel is then fixed at $K^{\prime}=K / P=5$. The subcarriers were randomly selected to form a subchannel using the diversity subcarrier method.

The channels between the CRs and the FC were modeled by independent and identically distributed impulse responses simulating 20, 25, 30, 40 and 75 propagation paths, respectively, under slow Rayleigh fading (constant during the sensing interval and independent from one sensing interval to the next). The numbers of propagation paths were chosen to match the number of OFDMA subcarriers so that a fast
Fourier transform (FFT) of the impulse response results in the $20,25,30,40$ or 75 coefficients that represent the channel gains for the subcarriers in the frequency domain. This setup simulates a frequency-selective Rayleigh fading channel that is flat in each subcarrier. The second moment of the channel gains was normalized to 1 so that the average signal-to-noise ratio (SNR) is not affected by these gains, on average, from the primary transmitter to the CRs. The primary signal was transmitted with unitary average power and the SNR at the input of each $\mathrm{CR}$ was configured as $-10 \mathrm{~dB}$. This small SNR regime was chosen to represent a more degrading, but yet realistic situation from the perspective of the spectrum sensing performance. For instance, the IEEE 802.22 standard [10] requires that the presence of digital TV transmissions should be sensed with a probability of detection of 0.9 with a sensitivity of $-114 \mathrm{dBm}$ (averaged over $6 \mathrm{MHz}$ ), which may be translated into very low SNR levels. In each sensing interval, $N=60$ samples of the primary received signal were collected by each $\mathrm{CR}$ in each subcarrier frequency.

Two simulation scenarios were considered: in the first one it is made a comparison between the performances of the EVF and the DF, with the corresponding test statistics GLRT, MMED, MED and ED, under different error probabilities $P_{\mathrm{e}}$ in the reporting channels, but without channel coding. This first scenario supports the analysis of the sensitivity of each scheme to the reporting channel errors. In the second scenario, the repetition and the $\mathrm{BCH}$ codes were used to protect the decisions in the DF scheme, and it was assessed the amount of redundancy sufficient for approximating the performances of the EVF and the coded DF for each decision fusion rule and test statistic. This second scenario helps the analysis of the trade-off between performance and volume of data over the reporting channels.

The receiver operating characteristic (ROC) curves presented hereafter were constructed from estimates of the probability of detection, $P_{\mathrm{d}}$, and the probability of false alarm, $P_{\mathrm{fa}}$, obtained from Monte Carlo simulations with 50000 runs for all sensed subchannels. The primary signal activity was modeled as a Bernoulli random variable simulating the ON state in $50 \%$ of the simulation runs (used when the probability of detection is estimated), and the OFF state in the other $50 \%$ (used when the probability of false alarm is estimated). The eigenvalues computed by each CR were digitized with a 4-bit resolution. This resolution has led to approximately the same performance achieved with full resolution (floating-point operation), and it is in close agreement with the resolution reported in [11].

\section{A. Results without channel coding}

Figs. 3-6 present ROC curves comparing the EVF and the DF schemes, respectively for the ED, the MED, the GLRT and the MMED techniques, without channel coding, under different values of $P_{\mathrm{e}}$. It can be noticed that, for $P_{\mathrm{e}}=0$, the EVF overcomes (higher $P_{\mathrm{d}}$ for a given $P_{\mathrm{fa}}$ or lower $P_{\mathrm{fa}}$ for a given $P_{\mathrm{d}}$ ) the DF in all cases shown, and that the ED achieves the better performance among the detection techniques, followed by the MED, the GLRT and the MMED. This ranking has also been verified in [8]. In terms of the 

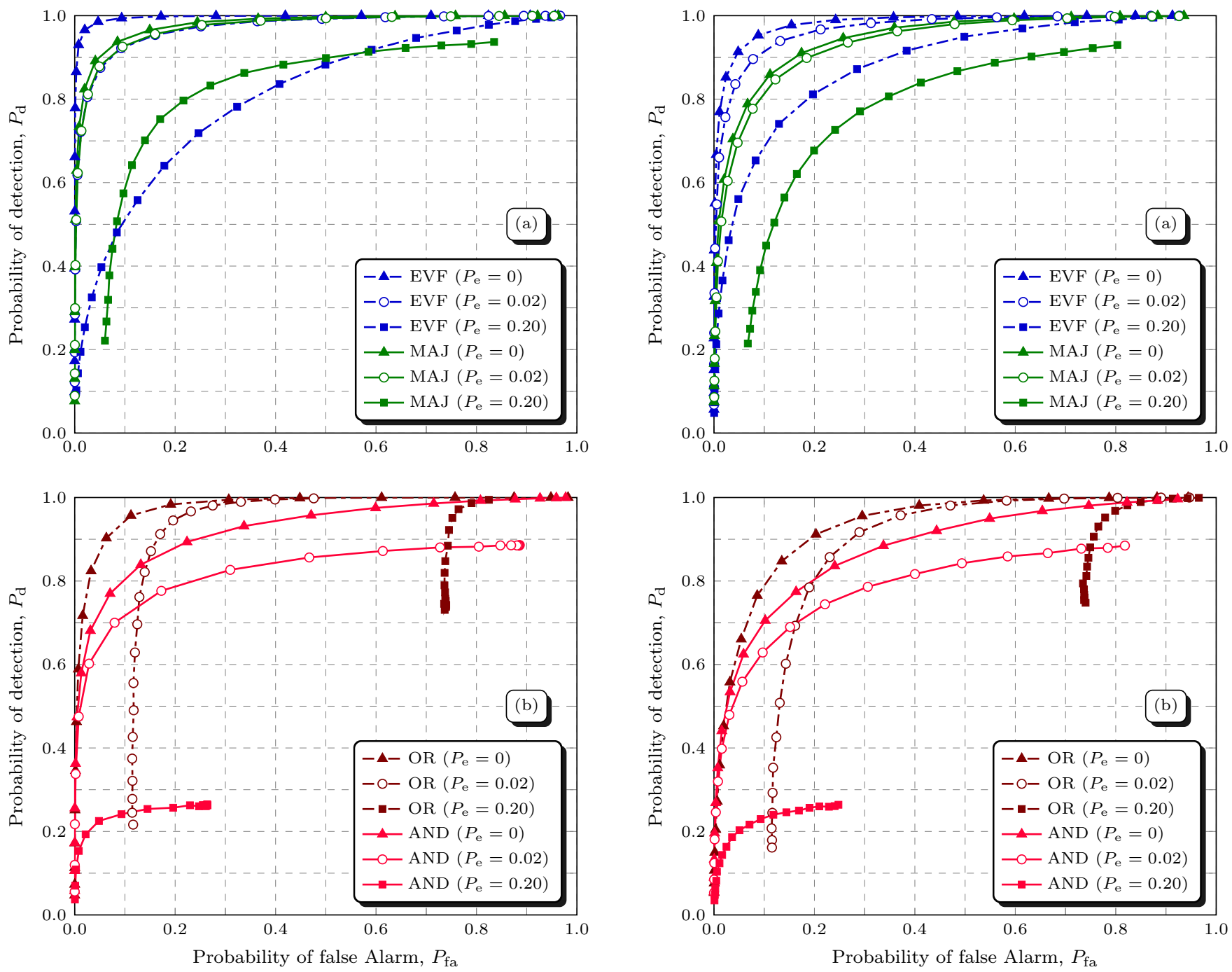

Fig. 3: ROCs for ED without channel coding and different $P_{\mathrm{e}}$ values for (a) EVF and DF MAJ, (b) OR and AND.

ranking of the DF schemes, the MAJ rule has produced the best performance, followed by the OR and the AND.

The performance degradation of all schemes and decision rules with the increase in $P_{\mathrm{e}}$ is also clearly observed in Figs. 3-6. The EVF again is superior, being less sensitive to the reporting channel errors than the DF rules. Among the DF rules, the MAJ has led to the lowest sensitivity to channel errors, followed by the OR and the AND rules. A technique with lower sensitivity to errors is defined here as the one for which, at a given $P_{\mathrm{fa}}$, the reduction in $P_{\mathrm{d}}$ is smaller than for the technique with higher sensitivity.

Also from Figs. 3-6 and from other ROCs presented later on, it can be observed that all DF rules can exhibit an expected saturation behavior in the $P_{\mathrm{fa}}$, in the $P_{\mathrm{d}}$ or in both as previously reported in [12] and [13]. For instance, $P_{\mathrm{fa}} \geq 1-\left(1-P_{\mathrm{e}}\right)^{m}$ for the OR rule, being this limit independent of the SNR [13]. Numerically, $P_{\text {fa }} \cong 0.11$ for the OR rule with the GLRT, under $P_{\mathrm{e}}=0.02$ (see Fig. 5), which is consistent with [13]. As the value of $P_{\mathrm{e}}$ increases, the bounds on $P_{\mathrm{fa}}$ or $P_{\mathrm{d}}$ become more
Fig. 4: ROCs for MED without channel coding and different $P_{\mathrm{e}}$ values for (a) EVF and DF MAJ, (b) OR and AND.

evident in the case of the OR and the AND rules than in the case of the MAJ.

The conjecture stated in [8] can be verified in part from Figs. 3-6: indeed, the DF scheme with the rules AND and OR are more sensitive to the reporting channel errors than the EVF scheme. However, the DF scheme with the MAJ rule is less sensitive to channel errors than the EVF only under low $P_{\mathrm{e}}$ regimes: for example, when $P_{\mathrm{e}}$ increases from 0 to 0.02 , the performance degradation is smaller in the DF scheme with the MAJ rule than in the EVF scheme; at higher values of $P_{\mathrm{e}}$, the bounds on $P_{\mathrm{d}}$ and $P_{\mathrm{fa}}$ start to become more pronounced in the DF with the MAJ rule, evidencing the lower sensitivity of the EVF in this situation.

A careful look in the graphs marked with (a) in Figs. 3-6, under the regimes of high $P_{\mathrm{e}}$, leads to the observation that the ED and the MMED unveiled a distinct behavior around the middle of their ROCs (around $P_{\mathrm{fa}}=1-P_{\mathrm{d}}$ ) in comparison with the GLRT and MED. Specifically, the DF scheme with the MAJ rule and detection techniques ED and the MMED has 

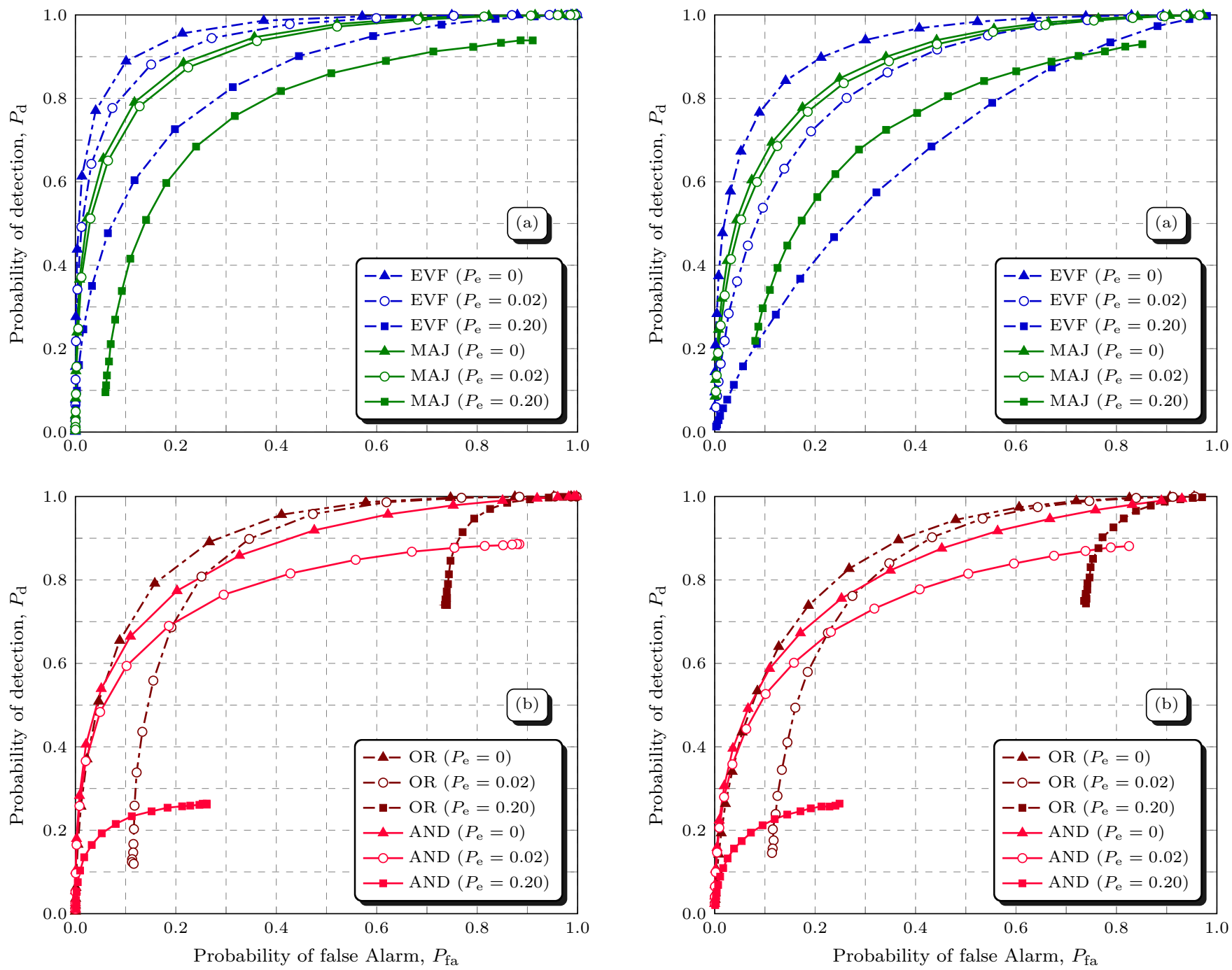

Fig. 5: ROCs for GLRT without channel coding and different $P_{\mathrm{e}}$ values for (a) EVF and DF MAJ, (b) OR and AND.

exhibited less sensitivity to reporting channel errors for high $P_{\mathrm{e}}$ than in the case of the GLRT and the MED. Thus, the relative performance degradation of the DF with an increased $P_{\mathrm{e}}$ has occurred in a less pronounced way. Nevertheless, in spite of the apparent superiority of the DF scheme with the MMED and the ED, the saturation effect of all decision fusion strategies renders the EVF as the better choice for the analyzed conditions.

\section{B. Results with channel coding}

Figs. 7-10 were generated to support the analysis of the trade-off between performance and volume of traffic over the reporting channels. The following procedure was followed to construct the ROC curves: the probability of error in the reporting channels, $P_{\mathrm{e}}$, was increased up to the value that shifts the EVF performance to approximately the same performance of the corresponding uncoded DF scheme with $P_{\mathrm{e}}=0$. As a consequence, for that value of $P_{\mathrm{e}}>0$ the performance of the uncoded DF scheme became worse (lower $P_{\mathrm{d}}$, higher $P_{\mathrm{fa}}$

Fig. 6: ROCs for MMED without channel coding and different $P_{\mathrm{e}}$ values for (a) EVF and DF MAJ, (b) OR and AND.

or both), as already shown in Subsection IV-A. Then, channel coding was enabled for the DF scheme, and the coding rate $r=k / n$ was progressively reduced (the redundancy $n-k$ was progressively increased) up to the point in which the performances of the coded DF and the uncoded EVF became approximately equal to one another.

Notice that all ROC curves presented in Figs. 7-10 consider both the $\mathrm{BCH}$ and the repetition code, except in Fig. 10(a), where only a result for the $\mathrm{BCH}$ code is shown. This is because the performance of the EVF reached the performance of the DF scheme with the MAJ rule and MMED, for $P_{\mathrm{e}}=0.0075$, before the channel errors have caused any significant degradation in the performance of the DF scheme, meaning that no redundancy was needed to approximate the corresponding performances in the case of the repetition code. Nevertheless, in this particular figure it has been inserted a result with a $\mathrm{BCH}$ code with the highest possible coding rate (smallest possible redundancy) so that the volume of traffic can be measured.

Each $\mathrm{BCH}$ code has been chosen as the one that was capable of resulting in a performance similar to that achieved with a 
repetition code with the smallest code rate $r=1 / n$, for each DF rule (MAJ, OR and AND). For example, it can be noticed in Fig. 9(b) that the performance obtained by the DF rule OR using the $\mathrm{BCH}$ code with $r=6 / 31$ is approximately equal to the one obtained with a repetition code with $r=1 / 13$.

One can also observe in Figs. 7-10 that results are shown for repetition codes, each one having approximately the same code rate, that is, approximately the same amount of redundancy bits per uncoded decision bit as the corresponding BCH code. For example, notice that in Fig. 9(b) there is also a result for the repetition code with $r=1 / 5$, and that $1 / 5 \cong 6 / 31 \cong 0.2$. Then, by considering approximately the same code rate for a $\mathrm{BCH}$ and a repetition code it is possible to establish a fair comparison in terms of performance.

The ROC curves in Fig. 9 show results for the EVF and the DF scheme under the rules MAJ, OR and AND, for the test statistic GLRT. In this case, the MAJ rule has achieved the best performance among the DF combining rules. Observing Fig. 9(a), it can be noticed that to achieve the same, or approximately the same performance of the EVF, the repetitioncoded DF scheme with the MAJ rule needs only $n=3$ bits to represent each uncoded CR decision per subchannel, against 13 with the OR rule, shown in Fig. 9(b), and 11 with the AND rule, shown in Fig. 9(c). Now considering the coded DF scheme when the MMED test statistic is used, as shown in Fig. 10, one can observe that approximate performances with respect to the EVF were obtained with $n=1,5,7$ for the rules MAJ, OR and AND, respectively. In the case of the coded DF scheme when the MED test statistic, it can be seen from Fig. 8 that approximate performances with respect to the EVF were obtained with $n=3,11,23$ for the rules MAJ, OR and AND, respectively. For the coded DF scheme with the ED, the number of bits used to represent each uncoded decision bit per subchannel were $n=3$ (MAJ), 9 (OR), and 11 (AND).

From above one can conclude that the MMED is the one that requires the smallest redundancy per decision bit and, thus, the smallest volume of traffic over the reporting channel, for all combining rules of the repetition-coded DF schemes. In the case of the $\mathrm{BCH}$-coded DF schemes, the MMED requires a smaller redundancy per decision bit for the MAJ, OR and AND combining rules. Moreover, $\mathrm{BCH}$-coded DF schemes with the MAJ rule have achieved the smallest, yet the same redundancy per decision bit for all test statistics (GLRT, MMED, MED and $\mathrm{ED})$.

Moreover, taking for example the GLRT, it can be observed from Fig. 9 that the $\mathrm{BCH}$-coded system overcomes the repetition-coded system when the DF schemes use approximately the same number of redundant bits per uncoded decision. In other words, the BCH code achieves in this case a larger error correction capability than the repetition code, both yielding approximately the same data traffic over the reporting channel during the transmissions of the coded CRs' decisions to the FC. If the MMED, the MED and the ED are analyzed under the same perspective of this paragraph, from Figs. 7, 8 and 10 one can draw the same conclusions obtained in the case of the GLRT, i.e. the BCH-coded DF schemes outperforms their repetition-coded counterparts for approximately the same number of redundant bits per uncoded CR decision.
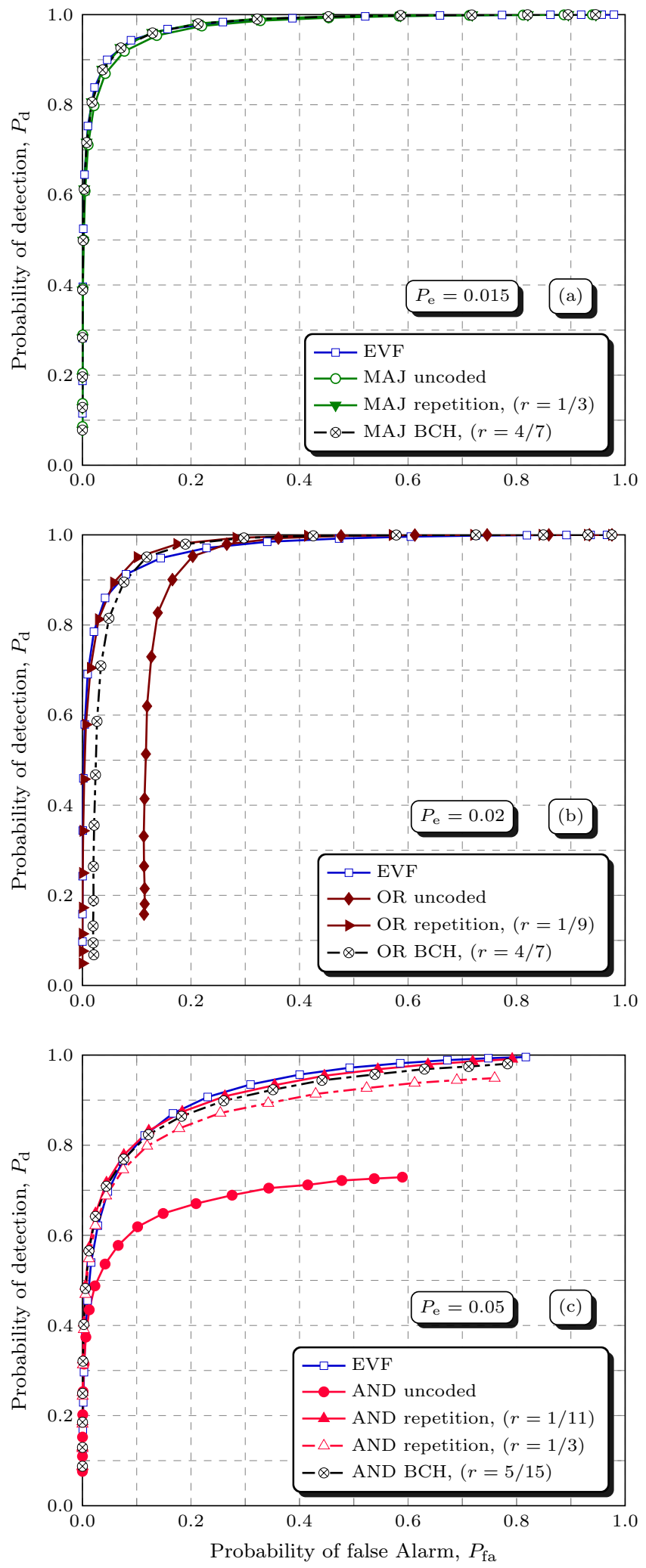

Fig. 7: ROCs for ED with channel coding for (a) EVF and DF MAJ, (b) OR and (c) AND.

A specific result is worth emphasizing here: from Fig. 8, for the coded DF schemes with MED and AND combining rule, it can be noticed that the performance obtained with 

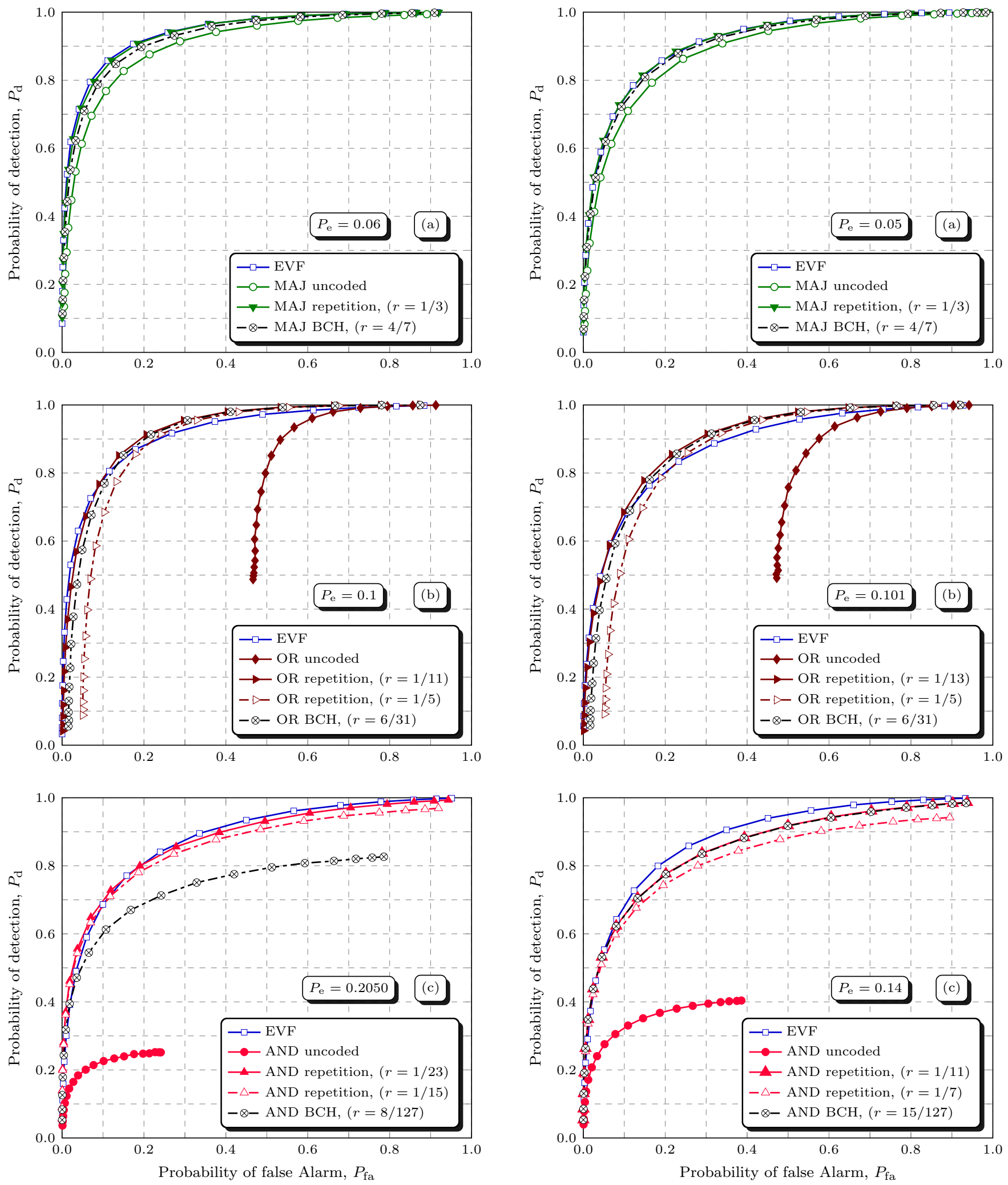

Fig. 8: ROCs for MED with channel coding for (a) EVF and DF MAJ, (b) OR and (c) AND.

the $\mathrm{BCH}(n, k, t)=(127,8,31)$ code, which is the best code configuration, did not even reach the performance yielded by the repetition code $(n, k, t)=(15,1,7)$. This is credited to

value of $P_{\mathrm{e}}=0.2050$, which is high when compared with the other tested values. This is meant to say that, in such a case, the average number of errors introduced by the channel 

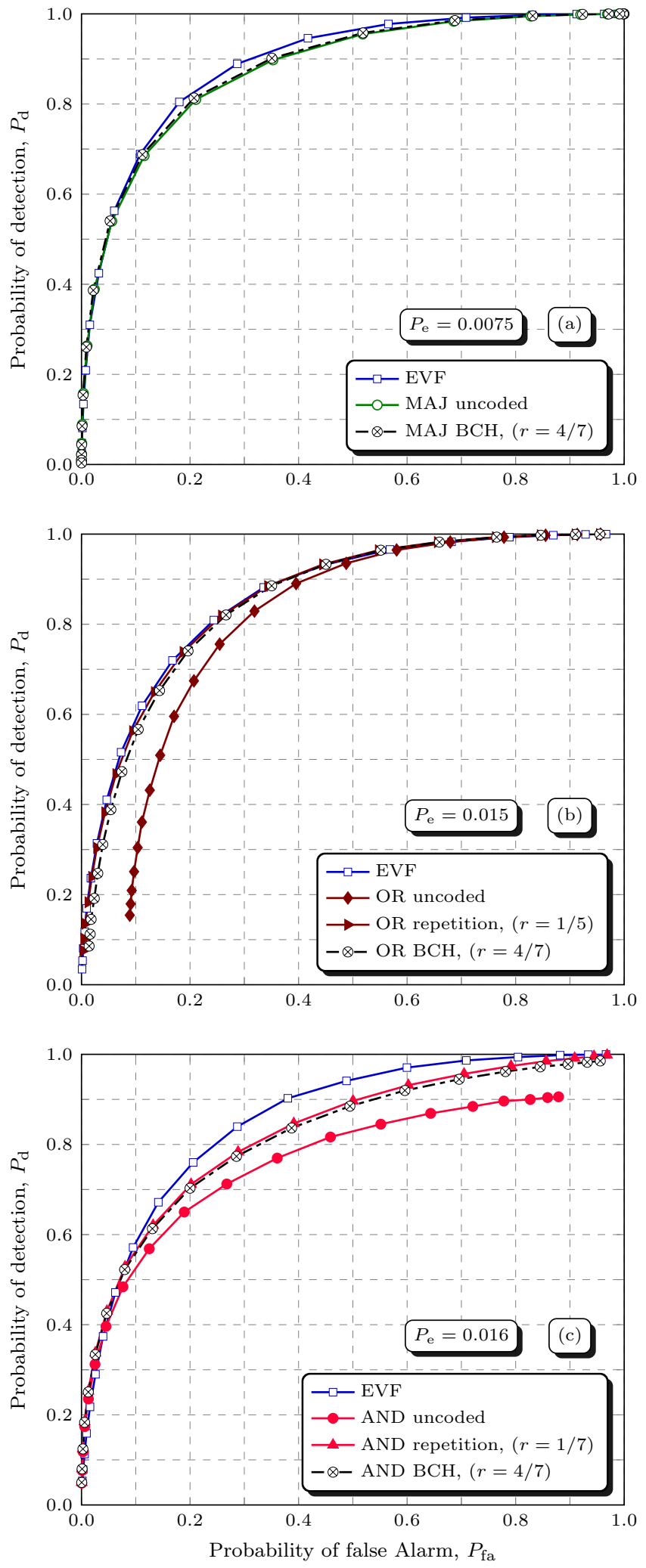

Fig. 10: ROCs for MMED with channel coding for (a) EVF and DF MAJ, (b) OR and (c) AND.

has exceeded the error correction capability of the $\mathrm{BCH}$ code. The probability that the number of errors $E$ exceeds the error correction capability $t$ of a block code can be computed from
[14, p. 323]:

$$
P_{\mathrm{r}}[E>t]=\sum_{i=t+1}^{n}\left(\begin{array}{c}
n \\
i
\end{array}\right) P_{\mathrm{e}}^{i}\left(1-P_{\mathrm{e}}\right)^{n-i} .
$$

Numerically, for the repetition code $(15,1,7), P_{\mathrm{r}}=0.005$. For the $\mathrm{BCH}$ code $(127,8,31), P_{\mathrm{r}}=0.1164$. Then, the probability that the number of errors introduced by the channel exceeds the error correction capability of the $\mathrm{BCH}$ code is 23.28 times larger than in the case of the repetition code.

Table I summarizes the main results obtained from all the presented ROC curves. It has been organized as follows: the first part, identified as Repetition code I, shows the pairs of values $\left(P_{\mathrm{e}} ; r\right)$ considering the repetition code with smallest code rate and the values of $P_{\mathrm{e}}$ responsible for pushing the performance of the EVF to approximately the same performance of the corresponding uncoded DF scheme with $P_{\mathrm{e}}=0$ (recall more details from Subsection IV-B). The parts identified as Repetition code II and BCH code also give the pair $\left(P_{\mathrm{e}} ; r\right)$, with the same values of $P_{\mathrm{e}}$ given before, considering those $\mathrm{BCH}$ codes that led to approximately the same performance of the repetition codes listed in the part Repetition code $\mathbf{I}$. However, the part identified as Repetition code II considers those repetition codes having approximately the same code rate of the corresponding $\mathrm{BCH}$ codes, that is, these repetition codes have approximately the same number of redundancy bits per uncoded decision bit as the associated $\mathrm{BCH}$ codes.

TABLE I: Bit error probability and coding rate, $\left(P_{\mathrm{e}} ; r\right)$, for each code type, test statistic and combining rule.

\begin{tabular}{llll}
\multicolumn{4}{c}{ Repetition code I } \\
\hline \hline & MAJ & OR & AND \\
\hline \hline GLRT & $(0.0500 ; 1 / 3)$ & $(0.1010 ; 1 / 13)$ & $(0.1400 ; 1 / 11)$ \\
MMED & $(0.0075 ; 1)$ & $(0.0150 ; 1 / 5)$ & $(0.0160 ; 1 / 7)$ \\
MED & $(0.0600 ; 1 / 3)$ & $(0.1000 ; 1 / 11)$ & $(0.2050 ; 1 / 23)$ \\
ED & $(0.0150 ; 1 / 3)$ & $(0.0200 ; 1 / 9)$ & $(0.0500 ; 1 / 11)$ \\
\hline \hline
\end{tabular}

\begin{tabular}{llll}
\multicolumn{4}{c}{ Repetition code II } \\
\hline \hline & MAJ & OR & AND \\
\hline \hline GLRT & $(0.0500 ; 1)$ & $(0.1010 ; 1 / 5)$ & $(0.1400 ; 1 / 7)$ \\
MMED & $(0.0075 ; 1)$ & $(0.0150 ; 1)$ & $(0.0160 ; 1)$ \\
MED & $(0.0600 ; 1)$ & $(0.1000 ; 1 / 5)$ & $(0.2050 ; 1 / 15)$ \\
ED & $(0.0150 ; 1)$ & $(0.0200 ; 1)$ & $(0.0500 ; 1 / 3)$ \\
\hline \hline
\end{tabular}

\begin{tabular}{llll}
\multicolumn{4}{c}{ BCH code } \\
\hline \hline & MAJ & OR & AND \\
\hline \hline GLRT & $(0.0500 ; 4 / 7)$ & $(0.1010 ; 6 / 31)$ & $(0.1400 ; 15 / 127)$ \\
MMED & $(0.0075 ; 4 / 7)$ & $(0.0150 ; 4 / 7)$ & $(0.0160 ; 4 / 7)$ \\
MED & $(0.0600 ; 4 / 7)$ & $(0.1000 ; 6 / 31)$ & $(0.2050 ; 8 / 127)$ \\
ED & $(0.0150 ; 4 / 7)$ & $(0.0200 ; 4 / 7)$ & $(0.0500 ; 5 / 15)$ \\
\hline \hline
\end{tabular}

The number $B$ of bits sent to the FC by each CR during the reporting of the CRs' decisions is directly proportional to the number of OFDMA subchannels for both the DF and the EVF schemes. As a consequence, the corresponding constant of proportionality can be removed from the trade-off analysis involving sensing performance and traffic over the reporting channels. For the EVF, $B$ is directly proportional to the order of the covariance matrix and to the number of quantization bits (the resolution) used for digitizing the eigenvalues, i.e. it is a number proportional to $K^{\prime} b=5 \times 4=20$ bits per 
CR (recall that the EVF was not submitted to channel coding in our analysis). For the coded DF schemes, $B$ is inversely proportional to the code rate. From the subtables Repetition code I and BCH code of Table I, one can see that, for the MAJ rule, $B$ is directly proportional to 1 and 1.75 for the MMED, and directly proportional to 3 and 1.75 for the GLRT, the MED and the ED. Considering the OR rule, $B$ is directly proportional to 13 and 5.17 for the GLRT, to 5 and 1.75 for the MMED, to 11 and 5.17 for the MED, and to 9 and 1.75 for the ED. From these results, it can be clearly noticed the reduction in $B$ for all cases if the $\mathrm{BCH}$ code is used, except the MMED with the MAJ rule and $P_{\mathrm{e}}=0.0075$, for which $r=1$, that is, no channel coding was needed to make the performances of the EVF and the DF close to each other.

From all the presented results, it can be concluded that the repetition-coded or the $\mathrm{BCH}$-coded $\mathrm{DF}$ schemes might be the preferred choices in terms of the number of bits sent to the FC by each CR during the reporting of the CRs' decisions, in spite of their higher sensitivity to the channel errors, except for the MED with the AND rule. In this specific case, if the repetition code is adopted, the value of $B$ was even greater than the corresponding one achieved by the EVF. Still referring to this specific case, but if the $\mathrm{BCH}$ code is adopted, the desired performance of the coded DF scheme was not met due to the high $P_{\mathrm{e}}$. As far as the remaining test statistics are concerned, i.e. the MMED, the GLRT and the ED, the BCH code is the best option for the DF scheme, since is possible to reduce the traffic over the reporting channels without sacrificing the performance relative to the one obtained with the repetition code. For instance, for the best situation with the ED and AND rule, the traffic is approximately 3 times smaller. Last but not least, the superiority of the MAJ rule is apparent, mainly for the MMED, for which the value of $P_{\mathrm{e}}=0.0075$ that produced comparable performances of the EVF and the DF did not lead to any significant performance degradation in the DF scheme.

\section{CONCLUSiOns}

In this paper it was analyzed the trade-off between the performance and the number of bits reported to the fusion center in centralized cooperative spectrum sensing techniques based on coded decision fusion and eigenvalue fusion strategies, under errors in the reporting channels. The analysis was carried out in the context of cognitive radio applications, considering that the primary network adopts orthogonal frequency division multiple access. It was verified that the bits that carry the decisions of the secondary (cognitive) users to the fusion center are more sensitive to the reporting channel errors than those used to represent the digitized eigenvalues in the eigenvalue fusion scheme. However, the amount of redundancy inserted by repetition encoding to protect the cognitive users' decisions and approximate the performance of the eigenvalue fusion scheme not always leads to a larger number of reported bits. Nevertheless, as demonstrated by the exemplifying results for $\mathrm{BCH}$-coded decision fusion schemes, the error correction capability of the adopted code has a large influence both in the performance of the spectrum sensing technique and in the net volume of traffic over the reporting channels. Although not always, the decision fusion schemes demonstrated advantages in terms of traffic over the reporting channel, whereas the eigenvalue fusion demonstrated advantages in terms of performance of the spectrum sensing. Then, as a general rule, the choice between them must be made via a trade-off analysis: if the main goal is performance, the choice has to made in favor of the eigenvalue fusion. Moreover, one must notice that the bits representing the eigenvalues in the eigenvalue fusion can be also coded, leading to a performance level that the coded decision fusion could not even reach. If the volume of traffic over the reporting channel is more important, the choice has to be shifted to the decision fusion. However, a clear choice might not be possible in all situations, and a case-by-case analysis is needed.

\section{REFERENCES}

[1] L. S. Costa, D. A. Guimarães, and R. A. A. de Souza, "Desempenho e tráfego sob o efeito da codificaçao de bloco nas fusões de decisões e de autovalores para sensoriamento espectral de sinais OFDMA," XXXIII Simpósio Brasileiro de Telecomunicações (SBrT 2015), pp. 1-5, Sep. 2015.

[2] J. Mitola III and G. Q. Maguire Jr., "Cognitive radio: making software radios more personal," IEEE Personal Commun. Mag., vol. 6, no. 4, pp. 13-18, Aug. 1999, doi: 10.1109/98.788210.

[3] T. Yucek and H. Arslan, "A survey of spectrum sensing algorithms for cognitive radio applications," IEEE Commun. Surveys Tuts., vol. 11, no. 1, pp. 116 -130, First 2009, doi: 10.1109/SURV.2009.090109.

[4] (2014, Jan.). [Online]. Available: http://standards.ieee.org/news/2014/ ieee_802_22_sos_study_group.html\#sthash.NtA6FtPc.dpuf

[5] N. Michailow, R. Datta, S. Krone, M. Lentmaier, and G. Fettweis, "Generalized frequency division multiplexing: A flexible multi-carrier modulation scheme for 5th generation cellular networks," in Proceedings of the German Microwave Conference (GeMiC'12), 2012.

[6] Z. Quan, S. Cui, A. H. Sayed, and H. V. Poor, "Optimal multiband joint detection for spectrum sensing in cognitive radio networks," IEEE Trans. Signal Process., vol. 57, no. 3, pp. 1128 -1140, Mar. 2009, doi: 10.1109/TSP.2008.2008540

[7] B. Nadler, F. Penna, and R. Garello, "Performance of eigenvaluebased signal detectors with known and unknown noise level," in IEEE Int. Conf. Communications, Jun. 2011, pp. 1-5, doi: 10.1109/icc.2011.5963473.

[8] D. A. Guimarães, C. R. N. da Silva, and R. A. A. de Souza, "Cooperative spectrum sensing using eigenvalue fusion for OFDMA and other wideband signals," Journal of Sensor and Actuator Networks, vol. 2, no. 1, pp. 1-24, 2013, doi: 10.3390/jsan2010001.

[9] D. A. Guimarães, L. S. Costa, and R. A. A. de Souza, "Comparison between eigenvalue fusion and decision fusion for spectrum sensing of OFDMA signals under errors in the control channel," in Telecommunications Symposium (ITS), 2014 International, Aug. 2014, pp. 1-5, doi: 10.1109/ITS.2014.6947981.

[10] IEEE Standard for Information Technology-Telecommunications and information exchange between systems Wireless Regional Area Networks (WRAN)-Specific requirements Part 22: Cognitive Wireless RAN Medium Access Control (MAC) and Physical Layer (PHY) Specifications: Policies and Procedures for Operation in the TV Bands, Jan. 2011.

[11] D. A. Guimarães and R. A. A. de Souza, "Implementation-oriented model for centralized data-fusion cooperative spectrum sensing," IEEE Commun. Lett., Nov. 2012, doi: 10.1109/LCOMM.2012.092112.121614.

[12] S. Chaudhari, J. Lunden, and V. Koivunen, "BEP walls for collaborative spectrum sensing," in IEEE Int. Conf. Acoustics, Speech and Signal Processing (ICASSP), May 2011, pp. 2984-2987, doi: 10.1109/ICASSP.2011.5946285.

[13] K. B. Letaief and W. Zhang, "Cooperative communications for cognitive radio networks," Proc. IEEE, vol. 97, no. 5, pp. 878-893, May 2009, doi: 10.1109/JPROC.2009.2015716.

[14] B. Sklar, Digital Communications: Fundamentals and Applications. Upper Saddle River, NJ, USA: Prentice-Hall, Inc., 2001. 


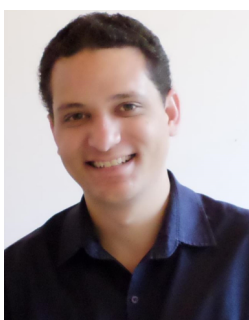

Lucas dos Santos Costa Lucas dos Santos Costa was born in Brazil in 1982. He received the B.S.E.E. and the M.Sc. degrees from the National Institute of Telecommunications, INATEL, in 2011 and 2014, respectively. Since 2015 he has been working at the Reference Center for Radiocommunications, whose research is concentrated on the fifth-generation $(5 \mathrm{G})$ of wireless and mobile communications. His main area of interest is related to digital communication systems and, specifically, he has been working with spectrum sensing for cognitive radio applications.



Dayan Adionel Guimarães was Born in Carrancas, MG, Brazil, on March 01, 1969. He holds the titles: Electronics Technician (ETE FMC, 1987), Electrical Engineer (Inatel, 1994), Specialist in Data Communication Engineering (Inatel, 2003), Specialist in Human Resources Management (FAI, 1996), Master in Electrical Engineering (Unicamp, 1998) and Doctor in Electrical Engineering (Unicamp, 2003). In 2010 he attended a Pos-Doctoral internship at Federal University of Santa Catarina (UFSC), studying Convex Optimization applied to telecommunications. From 1988 to 1993 he developed equipment for Industrial Instrumentation and Control, and also occupied the positions of Manufacturing and Product Engineering Supervisor at SENSE Sensores e Instrumentos. Since January 1995 he is Professor at Inatel where, for eight years, he was responsible for the structure that supports practical teaching activities for the Electrical Engineering undergraduate course. His research includes the general aspects of Digital Transmission and Mobile Communications.

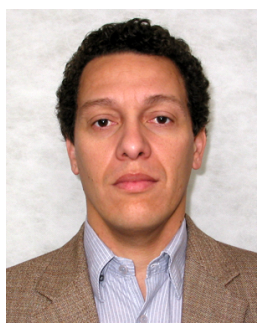

Rausley Adriano Amaral de Souza was born in Brazil in 1972. He received the B.S.E.E. and the M.Sc. degrees from the National Institute of Telecommunication, INATEL, in 1994 and 2002, respectively, and the Ph.D. degree from School of Electrical and Computer Engineering of the State University of Campinas, UNICAMP, Brazil. From 1995 to 2001, he worked as a Purchase Manager at Leucotron Equipamentos Ltda. He joined the National Institute of Telecommunication, INATEL, in 2002, where he is a Full Professor. He is presently a Researcher of the CNPq. Since 2015 he has been working at the Radiocommunication Reference Center, whose researches are concentrated on the fifth-generation (5G) systems. He is a Member of the Brazilian Telecommunications Society (SBrT) and is a Member of the IEEE. His general research interests include wireless communications, fading channel modeling and simulation, diversity systems, and spectrum sensing in cognitive radio systems. 